\title{
Synthèse
}

\section{La révolution génomique concerne aussi le bovin}

\author{
Jean-François Hocquette ${ }^{1}$ \\ Hubert Levéziel $^{2}$ \\ Gilles Renand ${ }^{3}$ \\ Alain Malafosse ${ }^{4}$ \\ ${ }^{1}$ Institut national de la recherche \\ agronomique (Inra), \\ UR 1213, \\ Unité de recherches sur les herbivores, \\ Theix, \\ 63122 Saint-Genès-Champanelle \\ <hocquet@clermont.inra.fr> \\ ${ }^{2}$ Institut national de la recherche \\ agronomique (Inra), \\ Université de Limoges, \\ UMR 1061, \\ Unité de génétique moléculaire animale, \\ 123 , avenue $\mathrm{A}$. Thomas \\ 87060 Limoges cedex \\ <hubert.leveziel@unilim.fr> \\ ${ }^{3}$ Institut national de la recherche \\ agronomique (Inra), \\ UR 337, \\ Station de génétique quantitative \\ et appliquée, \\ 78352 Jouy-en-Josas \\ <ugengre@dga2.jouy.inra.fr> \\ 4 Union nationale des coopératives agricoles \\ d'élevage et d'insémination animale \\ (UNCEIA), \\ 149, rue de Bercy, \\ 75595 Paris cedex 12 \\ <Alain.Malafosse@unceia.fr>
}

\begin{abstract}
Résumé
La génomique est une véritable révolution de la biologie aux nombreuses applications dans toutes les sciences de la vie. Le séquençage des génomes de différentes espèces, ainsi que les techniques d'étude du polymorphisme et de l'expression des gènes représentent une avancée supplémentaire vers la compréhension des fonctions biologiques des gènes. Dans le cadre de cette évolution, l'expertise des physiciens et des informaticiens se combine avec celle des généticiens et des physiologistes pour mieux comprendre comment les gènes contrôlent la dynamique des fonctions cellulaires. Les applications de la génomique sont naturellement les plus prometteuses pour l'espèce humaine dans le domaine médical. En effet, une médecine préventive et individuelle, car adaptée au potentiel génétique de chacun et à ses conditions de vie, va probablement se développer à moyen terme. En ce qui concerne les espèces agronomiques, nul doute que le séquençage du génome bovin apportera de nouveaux concepts et de nouveaux outils moléculaires qui accéléreront les recherches en génomique bovine. La comparaison du génome bovin avec celui d'autres espèces comme l'homme est aussi une source potentielle de progrès importants, que ce soit pour la santé humaine ou l'élevage des ruminants en général.
\end{abstract}

Mots clés : bovin ; génomique.

Thèmes : productions animales ; méthodes et outils.

\section{Abstract \\ The genomics revolution also applies to the bovine genome}

Genomics has brought about in its wake a true biological revolution and can be applied to all areas of life sciences. Having sequenced the genomes of several different species, genomics techniques now allow us to study polymorphisms and the gene expression of proteins, enhancing our understanding of the biological functions of genes. The combined know-how of physicists and computer scientists, as well as of geneticists and physiologists is required to identify how genes control the dynamics of cell functions. Naturally enough, it is in the sphere of human medicine that the applications of genomics look most promising. Preventive and customized medicine, adapted to the genetic potential and to the way of life of each individual, will in all probability see the light of day in the foreseeable future. In farm animals, there is no doubt that sequencing the bovine genome will give rise to new concepts and new molecular tools which will accelerate studies in bovine genomics. Comparing the bovine genome with that of other species, including humans, is also a potential source of huge progress for both human health and ruminant husbandry in general.

Key words: cattle; genomics.

Subjects: animal productions; tools and methods.

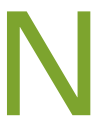
ous avons vécu au $\mathrm{xx}^{\mathrm{e}}$ siècle une révolution dans les sciences de la communication (radio, télévision, informatique, réseau Internet, téléphone portable, etc.). Nous sommes en train de vivre en ce début de $\mathrm{XxI}^{\mathrm{e}}$ siècle une véritable révolution en biologie qui est porteuse d'espoir pour toutes les sciences de la vie, notamment en médecine, pharmacologie et, pour ce qui nous concerne ici, la filière bovine. 
Compte tenu des enjeux de santé publique liés aux maladies de notre société moderne (cancer, obésité, diabète, Alzheimer, etc.) et en raison de l'importance des investissements possibles dans ce domaine, les sciences médicales (cancérologie, neurosciences, pharmacologie, toxicologie, etc.) sont généralement les premières à exploiter les progrès en sciences de la vie. C'est pourquoi, elles n'hésitent pas à utiliser au moins en partie les progrès scientifiques et technologiques issus de la génomique.

En revanche, les sciences animales ont toujours eu plus de difficultés à suivre les progrès de la biologie. Ainsi, avec des moyens financiers beaucoup plus limités que ceux disponibles pour la santé humaine, les filières animales sont amenées à effectuer des arbitrages stratégiques et financiers importants entre recherche fondamentale et applications finalisées des progrès de la recherche. Les acteurs de ces filières sont, de plus, confrontés à la fois à un contexte socioéconomique changeant, de plus en plus complexe, et à une recherche scientifique en pleine mutation. Le rythme des découvertes qui s'accélère sans cesse, des connaissances de plus en plus approfondies sur le vivant, un nombre croissant de biotechnologies novatrices et une masse d'informations biologiques (en génétique ou en physiologie) qui inondent les acteurs des filières sont des phénomènes que nous connaissons tous.

Cet article se propose donc de faire un état des lieux des progrès en génomique et des perspectives futures dans ce domaine, notamment en ce qui concerne les bovins afin d'éclairer les acteurs de cette filière.

\section{Qu'est ce que la génomique?}

\section{Les progrès de la biologie}

Nous ne retracerons pas ici les grandes étapes de la génétique et de la biologie moléculaire depuis la découverte des lois de l'hérédité (1866) par Mendel jusqu'à la mise en évidence de la structure de l'ADN (1953) par Crick et Watson et le développement des techniques de biologie moléculaire à partir des années 1960-1970 (http://www.genoscope.cns.fr/externe/ HistoireBM) qui ont permis des avancées considérables dans notre connaissance des génomes et de l'expression des gènes. Le lecteur est invité à se référer à différents articles sur le sujet concernant par exemple la structure de l'ADN et des chromosomes (Hayes, 2000), les grandes étapes de l'utilisation de l'information génétique (Leroux et Tosser-Klopp, 2000), la cartographie des génomes complexes (Cribiu et Schibler, 2000), etc. Nous nous attacherons dans cet article à préciser ce qu'est la génomique aujourd'hui et ses perspectives à moyen et long terme.

La génomique ${ }^{1}$ (glossaire) est la science du génome (c'est-à-dire de l'ensemble des gènes). Cette science (qui n'est pas nouvelle) est en plein essor grâce au développement de techniques modernes de biotechnologie à la fois miniaturisées, automatisées et rapides. C'est pourquoi, il est question aujourd'hui de méthodes à "haut débit". En effet, des procédures automatisées accomplies par des robots permettent aujourd'hui de couper, copier, amplifier et séquencer ${ }^{2}$ l'ADN. Ces technologies rendent possible l'étude de milliers de gènes en même temps. Par ailleurs, des milliers de gènes peuvent être imprimés sur une surface aussi petite qu'un timbre de poste. Il s'agit de puces $^{3}$ à $\mathrm{ADN}$ qui reposent sur une synergie entre différentes disciplines comme la physique, l'électronique et la biologie. Toutes ces approches peuvent être comparées à de véritables laboratoires de recherche miniaturisés, dans la mesure où le travail réalisé il y a quelques années par une équipe de recherche conséquente peut l'être aujourd'hui par des robots contrôlés par une seule personne. Parties de l'ADN, ces approches à haut débit concernent de plus en plus toutes les molécules de la vie (acides nucléiques, protéines, métabolites ${ }^{4} . .$. ) (Hocquette, 2005). La mise en ouvre de la génomique nécessite donc un effort important pour acquérir la maîtrise technique des nouvelles biotechnologies.

La génomique se décompose classiquement en deux grands sous-ensembles : la génomique dite structurale et la génomique dite fonctionnelle.

La génomique dite structurale met en œuvre des approches de plus en plus fines pour baliser et séquencer le génome. L'étape ultime est la détermination de la structure des gènes et des polymorphismes ${ }^{5}$ qui ont un impact fort sur les caractéristiques des animaux. Ce travail est aujourd'hui facilité par le séquençage à haut débit. Après des virus, des bactéries, l'homme, le rat et plus récemment le bovin, le nombre d'organismes dont le génome est séquencé ou en cours de séquençage augmente de façon exponentielle (Hocquette, 2005) (figure 1).

La génomique dite fonctionnelle étudie l'expression ${ }^{6}$ des gènes sous forme de transcrits ${ }^{7}$ puis de protéines, molécules qui déterminent les caractéristiques des cellules, tissus et organismes. L'objectif est d'analyser l'ensemble des transcrits (le transcriptome) et des protéines (le protéome) présents à un instant donné dans les tissus ou les organes des êtres vivants. En effet, notre patrimoine génétique ne suffit pas à lui seul au déterminisme des caractères. C'est bien l'expression des gènes qui déterminera finalement la réalisation des caractères, ou phénotype ${ }^{8}$.

\section{$\mathrm{Nb}$ de génomes séquencés et publiés}

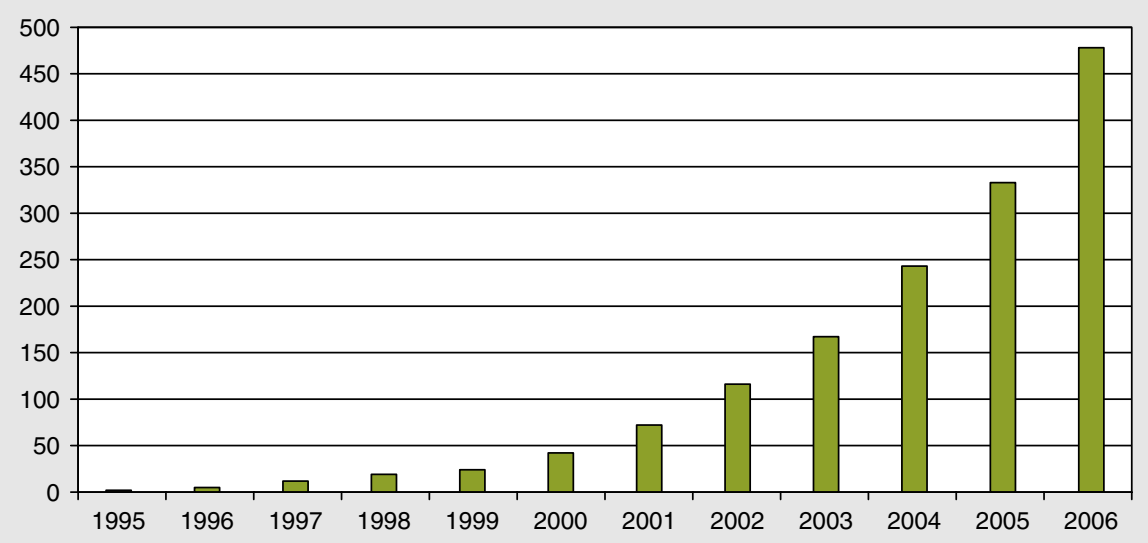

Figure 1. Évolution du nombre de génomes complètement séquencés de 1995 à décembre 2006.

Figure 1. Evolution of the number of completely sequenced genomes from 1995 to December 2006. Source : http://genomesonline.org/gold_statistics.htm 
Les caractéristiques des animaux changent avec le temps et l'environnement, alors que leur génotype reste constant. L'exemple le plus classique est le papillon qui ne ressemble en aucune façon à la larve dont il est issu. Et pourtant la larve et le papillon ont les mêmes gènes. Il nous faut donc considérer une vision dynamique de l'expression des gènes, et à un même génotype correspondent ainsi plusieurs transcriptomes, et plusieurs protéomes, en fonction du temps et de l'environnement. Aujourd'hui, après l'ADN, les transcrits et les protéines, le nouveau défi des biotechnologies modernes est le métabolome. Par analogie, il s'agit de l'ensemble des métabolites ${ }^{4}$ présents dans une cellule, un tissu, un organisme. Les techniques les plus utilisées pour identifier et doser les métabolites sont les techniques basées sur la spectrométrie de masse $e^{10}$. Le nombre de publications scientifiques sur le transcriptome et le métabolome augmente également de façon exponentielle, traduisant la révolution génomique qui affecte les sciences biologiques (figure 2). D'une façon générale, les biologistes ne sont pas à court d'imagination pour proposer de nouveaux termes tels que le sécrétome (ensemble des protéines sécrétées), le phosphoprotéome (ensemble des protéines phopshorylées), le glycogénome (ensemble des gènes impliqués dans les modifications posttraductionnelles des lipides et des protéines par les processus de glycosylation)... ces nouvelles identités apparaissant avec le développement des nouvelles technologies à haut débit qui permettent de les étudier de façon exhaustive (Hocquette, 2005).

\section{Le développement de la biologie intégrative et de la bioinformatique}

D’une façon générale, la nécessité de relier les gènes à la physiologie est reconnue depuis les débuts de la biologie qui, par essence, est dite "intégrative ". Son objectif est en effet de relier (c'est-à-dire "intégrer") toutes les connaissances sur les gènes aux mécanismes biologiques (Hocquette, 2005) pour mieux comprendre le fonctionnement des organismes vivants, les connaissances à "intégrer" pouvant être issues des recherches conduites sur de très nombreux modèles cellulaires ou animaux comme la souris. La nouveauté est que cette intégration est rendue probablement plus facile (ou en tout cas plus faisable) en raison de l'accumulation de données liées aux techniques à haut débit et en raison du développement de la bioinformatique.

Les techniques modernes de génomique permettent en effet la génération de quantités importantes de données biologiques à différents niveaux du vivant (ADN, transcrits, protéines, métabolites...) grâce à la puissance des techniques utilisées. Il est clair que la première étape de la biologie intégrative consiste à stocker et analyser les données générées par la génomique. Les chercheurs ont par conséquent besoin d'importantes puissances de calcul et de bases de données informatiques. C'est pourquoi, se déve-

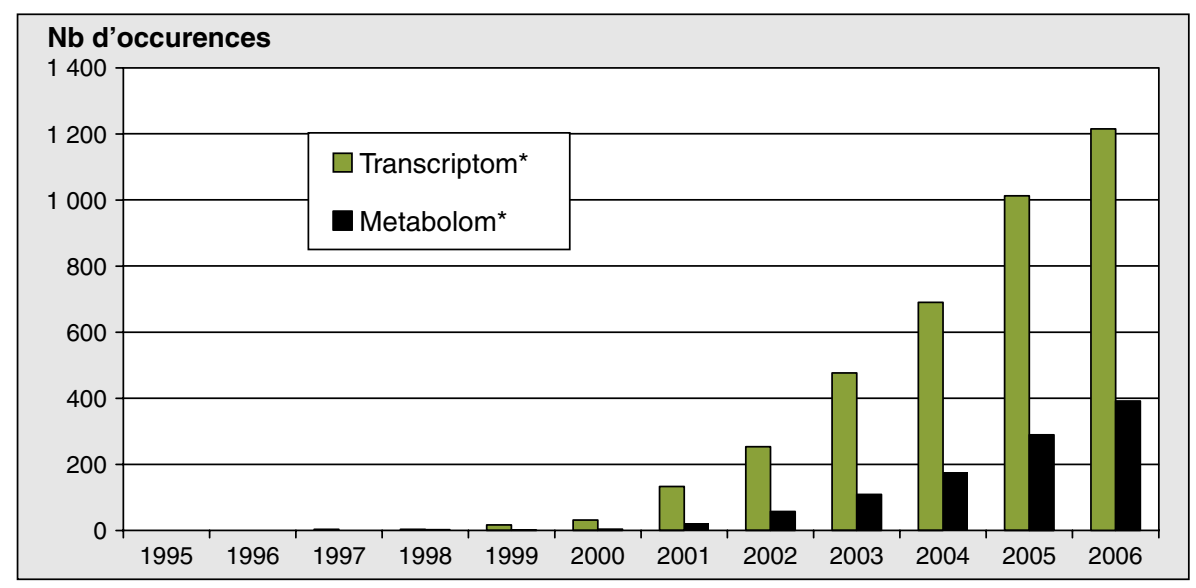

Figure 2. Nombre de publications scientifiques dans la base de données ISI Web of Science avec le terme " transcriptom* " (incluant transcriptome, transcriptomics, etc.) ou le terme " metabolom* " (incluant metabolome, metabolomics, etc.) dans le titre, les mots-clés ou l'abstract, entre 1995 et décembre 2006.

Figure 2. Number of scientific publications in the ISI Web of Science data base featuring the term "transcriptom*" (including transcriptome, transcriptomics, etc.) or the term "metabolom* " (including metabolome, metabolomics, etc.) in the title, the key words or the abstract, between 1995 and December 2006 . loppe conjointement à la génomique une autre science nouvelle, la bioinformatique, ou science de la gestion de l'information biologique. Son objectif est de "mettre le vivant en équations " pour donner un sens aux données biologiques. Les besoins en informatique sont aujourd'hui proportionnels à la capacité des chercheurs à étudier la complexité biologique des êtres vivants à partir de la connaissance de leur génome (Tuggle et al., 2006) (figure 3). L'ambition actuelle des bioinformaticiens est de construire des modèles biologiques qui permettraient de simuler l'organisation et la dynamique des cellules vivantes. Les projets de modélisation globale du fonctionnement des cellules concernent en premier les systèmes les plus simples que sont par exemple les bactéries (Rechenmann et de Jong, 2005).

Enfin, en raison de ces approches de biologie intégrative, les chercheurs peuvent avoir une vision globale de la fonction des gènes dans une cellule, un tissu ou un organisme. Il s'agit d'approche systémique car il est possible aujourd'hui d'étudier les systèmes biologiques dans leur ensemble. L'historienne américaine Evelyne Fox Keller nous explique que les gènes sont comme les acteurs d'une pièce de théâtre. De même que chaque acteur joue un rôle spécifique rarement interchangeable, chaque gène a une fonction biologique précise. Les recherches ont porté jusqu'à présent sur l'identification des gènes, donc sur l'identité des acteurs du théâtre de la vie. Aujourd'hui, la génomique permet de comprendre le scénario de la pièce de théâtre à partir de la fonction des gènes (le rôle des acteurs), c'est-à-dire comment les gènes (les acteurs) interagissent entre eux de façon dynamique pour déterminer les caractères physiologiques. De même qu'un groupe d'acteurs donné peut jouer plusieurs pièces de théâtre différentes, le même groupe de gènes peut conduire à des phénotypes différents selon son interaction avec le milieu (Fox Keller, 2004). En effet, la vie ne réside pas dans les gènes eux-mêmes, mais dans leur expression (interprétation du scénario) et leurs interactions en réseaux (relations entre les acteurs), qui sont également dépendantes de l'environnement. C'est pourquoi, la science des réseaux est porteuse d'avenir pour la biologie. Certains auteurs pointent judicieusement les analogies de "l'interactome" (ensemble des interactions entre molécules dans la cellule comme par exemple les interactions 


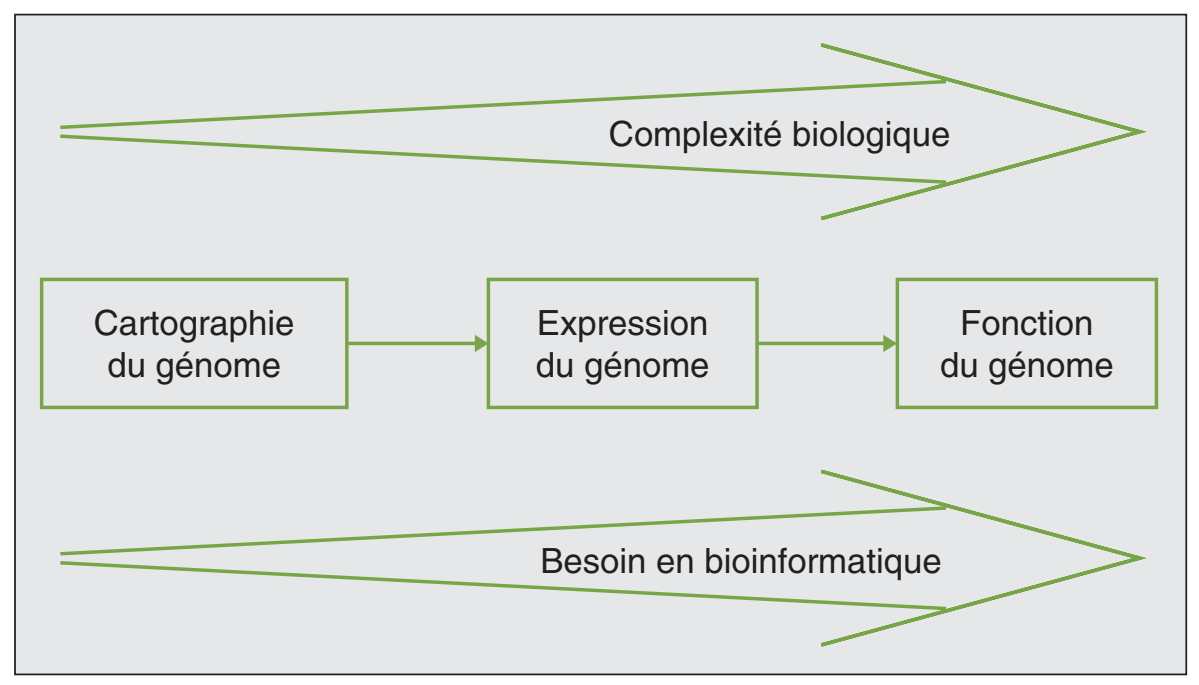

Figure 3. Évolution des recherches en génomique et en bioinformatique.

Figure 3. Schematics of research phases in genome biology and in bioinformatics.

Au fur et à mesure du développement de la génomique, les questions de recherche relatives au traitement des données deviennent plus complexes. Ainsi, l'intégration des données biologiques est de plus en plus dépendante donnees deviennent plus complexes. Ainsi, 1 'integra
des outils bioinformatiques (Tuggle et al., 2006).

protéines-protéines) avec par exemple l'architecture des circuits électroniques, le réseau Internet ou encore les réseaux des relations humaines observées en sciences sociales. Les mathématiques, la physique, l'informatique, les statistiques apportent donc de nouveaux outils pour mieux comprendre la biologie.

\section{Le séquençage du génome bovin et les outils moléculaires disponibles}

Comme indiqué précédemment, les applications des recherches en génomique chez l'homme représentent un enjeu médical majeur pour notre société. La génomique consiste ici à adapter le traitement médical au génome et aux conditions de vie du patient en se fondant sur les interactions entre les médicaments, les spécificités génétiques et le milieu de vie (alimentation, activité physique, stress, etc.) du patient. La génomique devrait aboutir à une forme de médecine individuelle et prédictive, donc nettement plus efficace. Mais plusieurs dizaines d'années peuvent s'écouler avant la généralisation de ces pratiques.

\section{Les apports du séquençage du génome bovin}

Les programmes de séquençage à haut débit, notamment celui du génome humain, apportent bien évidemment une masse d'informations précieuses à la compréhension des phénotypes physiologiques et pathologiques chez l'homme. Le séquençage du génome des organismes modèles a également contribué à cette meilleure compréhension. Mais il est clair que le génome d'autres espèces proches de l'homme est nécessaire pour mieux appréhender l'évolution génétique qui a conduit à l'apparition de l'espèce humaine, d'une part, et pour mieux appréhender la complexité du déterminisme des fonctions biologiques en utilisant des outils de génomique et de biologie comparatives, d'autre part.

Dans ce contexte, tout en n'étant ni un primate, ni un rongeur, l'espèce bovine figure parmi les mammifères placentaires typiques comme l'espèce humaine (Gibbs et al., 2004). Sur le plan de la différenciation musculaire au cours de la vie fotale, il a notamment été montré par l'Institut national de la recherche agronomique (Inra) que le bovin est une espèce mature à la naissance, comme l'homme et contrairement aux autres espèces animales étudiées dont le tissu musculaire est beaucoup moins différencié à la naissance (Picard et al., 2002). Le séquençage du génome bovin devrait donc être très informatif par rapport aux objectifs généraux de santé humaine (Gibbs et al., 2004). De plus, le bovin est une espèce d'intérêt agronomique importante, que ce soit au niveau mondial, européen ou français. Avec 1,33 milliard de têtes en 2004, l'espèce bovine est présente sur tous les continents pour la production de lait ou de viande. En termes d'effectifs mondiaux des espèces animales d'élevage, elle n'est devancée que par le poulet (16,2 milliards) dont le génome a depuis été séquencé, et suivie par le mouton (environ 1,04 milliard de têtes). Alors que le continent européen accueille seulement $10 \%$ du cheptel bovin mondial, il contribue à hauteur de 19,6 et 40,4\% respectivement à la production de viande bovine et de lait de vache, ce qui représente un enjeu économique majeur (données de 2004) (Rosati et al., 2005). La production bovine de lait et viande cumulée est en fait de loin le premier poste économique des productions animales en Europe. Dans le contexte européen, la France est le premier producteur de viande bovine et le deuxième producteur de lait de vache (Liinamo et al., 2003). La séquence du génome bovin servira d'outil aux chercheurs en élevage qui souhaitent améliorer la santé du bétail et la qualité sensorielle et nutritionnelle des produits laitiers et carnés, contribuant ainsi au développement durable de l'élevage (Harlizius et al., 2004). La comparaison du génome bovin avec d'autres génomes permettra aussi une meilleure connaissance du génome d'espèces proches comme le mouton ou la chèvre. L'étude du déterminisme génétique des caractères d'intérêt agronomique en sera ainsi facilitée chez les ruminants.

Enfin, sur le plan technique, la connaissance du génome bovin était très avancée et les outils moléculaires disponibles nombreux en 2003 (Gibbs et al., 2004).

L'ensemble de ces arguments (intérêt pour les sciences biomédicales et agronomiques, état de l'art scientifique et technique) a conduit à la mise en place d'un consortium qui a décidé de mobiliser 53 millions de dollars pour le séquençage du génome bovin. En raison de l'enjeu pour la santé humaine, le principal financeur (25 millions de dollars) est le National Human Genome Research Institute (NHGRI) qui fait partie du National Institute of Health (NIH) aux ÉtatsUnis. Contribuent également le département américain de l'agriculture (United States Department of Agriculture, USDA) (11 millions de dollars), l'État du Texas 
(8 millions de dollars), Genome Canada (5 millions de dollars), la Fondation Kleberg (2 millions de dollars), la Commonwealth Scientific and Industrial Research Organisation (CSIRO) en Australie (1 million de dollars), la NouvelleZélande (1 million de dollars), le United States Beef Council (0,4 million de dollars), le Texas Beef Council et le South Dakota Beef Council (<0,2 million de dollars) (Gibbs et al., 2004).

Le génome bovin est semblable par sa taille au génome de l'homme et autres mammifères : en effet, il contient 3 milliards de paires de bases d'ADN. Une équipe dirigée par le $\mathrm{Dr}$ R. Gibbs au Baylor College of Medicine à Houston a la responsabilité de son séquençage. Le premier bovin dont le génome est séquencé est celui d'une vache Hereford appelée "Dominette ". D'après les résultats publiés en août 2006, les efforts de séquençage aujourd'hui réalisés ont abouti à une couverture dite de 7,1 fois du génome bovin. Cela signifie que le volume des données acquises, équivalent au séquençage de plus de sept fois du génome total, permet par la redondance et les vérifications qui sont ainsi faites, d'établir la séquence du génome avec une qualité satisfaisante (http://www. hgsc.bcm.tmc.edu/projects/bovine/).

D'autres programmes de séquençage plus ciblés, ayant pour objectif de mettre en évidence des polymorphismes ${ }^{5}$ de type SNP (single nucleotide polymorphism), c'est-à-dire des mutations qui existent dans le génome de certains individus (Gibbs et al., 2004) ont été mis en place. Ils concernent actuellement des animaux de race Holstein, Angus, Jersey, Norwegian Red, Braham, et une seule race française, la Limousine, à propos de laquelle il convient de mentionner la contribution des organisations de la filière limousine pour le choix et la mise à disposition de l'ADN des animaux étudiés, et pour sa participation financière au travail de séquençage ainsi que celle de l'université de Limoges. Il doit être souligné que ces programmes sont en pleine phase de production et que plusieurs dizaines de milliers de SNP seront prochainement décrits et mis à disposition de la communauté internationale.

\section{Les puces à ADN bovines}

Parallèlement à ces efforts de séquençage, de nombreuses équipes de recherche se sont investies dans la préparation de collections de fragments d'ADNc partiellement séquencés permettant de pré- parer des puces à ADN pour l'étude de l'expression des gènes. Ces collections d'ADNc correspondent, par construction, à l'ensemble des gènes exprimés dans les organes ou tissus des animaux à partir desquels elles ont été construites. De telles collections ont été préparées pour le foie, l'intestin, l'embryon, l'endomètre, l'utérus, les ovaires... (Moody et al., 2003 ; Hocquette et al., 2005) de sorte que le nombre de séquences d'ADNc présentes dans les bases de données internationales dépasse actuellement 1400000 pour le bovin. La contribution la plus importante a été réalisée par le National Bovine Functional Genomics Consortium (NBFGC, États-Unis) qui a réalisé différentes collections d'ADNc à partir de plusieurs tissus bovins mélangés (Smith et al., 2001) dans le but de préparer des puces à ADN utilisables quel que soit le tissu (Suchyta et al., 2003). Des chercheurs coréens (Cho et al., 2002) et australiens (Lehnert et al., 2004) ont contribué à cet effort international en préparant des collections d'ADNc à partir d'un mélange de muscles et tissus adipeux bovins pour maximiser la probabilité d'étudier des gènes impliqués dans le développement musculaire et l'accumulation de lipides dans la viande (processus biologique conduisant à la production d'une viande persillée et ayant du goût). Différentes équipes françaises de l'Inra ont contribué à la mise en place de ces outils moléculaires en préparant plusieurs banques d'ADNc à partir de différents tissus ou organes pris individuellement, notamment de différents muscles qui présentent un intérêt pour les bovins à viande (Sudre et al., 2005), de l'endomètre, de l'embryon et de la glande mammaire, plusieurs de ces collections ayant été ensuite rassemblées sur les mêmes puces à ADN (Bernard et al., 2005).

Aujourd'hui, les progrès technologiques et la disponibilité d'un grand nombre de séquences d'ADNc bovines ont orienté ce domaine vers une évolution méthodologique consistant en la réalisation de puces à oligonucléotides. Le principe repose sur la sélection à l'aide d'outils bioinformatiques de séquences d'ADN courtes (ou oligonucléotides) qui sont très spécifiques des gènes ou parties de gènes étudiés. Cette nouvelle technologie est plus reproductible que la première et fait actuellement l'objet, chez le bovin, de puces commercialisées par des entreprises privées comme par exemple Affymetrix (http://www.affymetrix.com/ products/arrays/specific/bovine.affx) ou d'ensembles d'oligonucléotides prêts à être déposés sur des puces (Opéron). Nul doute que la disponibilité imminente de la séquence du génome bovin accélérera la conception de ce type d'outils, qui devraient devenir aisément accessibles à la plupart des laboratoires concernés par l'espèce bovine. Parallèlement et sans limitation d'espèce, la standardisation des pratiques en laboratoire, notamment à travers le protocole MAQC (microarray quality control) (Shi et al., 2006), assure aujourd'hui une bonne reproductibilité des résultats obtenus avec les puces à $\mathrm{ADN}$, et de ce fait, une comparaison fiable entre les résultats obtenus par les principales équipes internationales travaillant dans ce domaine (Canales et al., 2006).

\section{Conclusion}

Grâce au développement des techniques puissantes de génomique, nous serons probablement capables, dans le futur, de déterminer de façon automatisée et reproductible les polymorphismes de l'ADN ainsi que le niveau d'expression des gènes ou de leurs protéines dans les tissus. Les applications de la génomique les plus évidentes sont dans le domaine médical. Ainsi, par exemple, la médecine deviendra probablement préventive et individuelle. Elle sera en effet adaptée au potentiel génétique de chaque individu, ainsi qu'à ses conditions de vie et plus spécifiquement à son alimentation.

En ce qui concerne l'élevage bovin, ces outils permettront d'établir de nombreuses interactions entre les différents programmes de recherche portant par exemple sur la reproduction et la fertilité des femelles laitières ou allaitantes, la quantité et la nature des matières grasses dans le lait ou la viande, la qualité des produits, etc.

En conclusion, la mise en œuvre des approches de la génomique est engagée pour de nombreuses applications, notamment en médecine et en sciences animales. Clairement, le séquençage du génome bovin va donner un nouvel élan à ces recherches. C'est pourquoi, la génomique bovine est une priorité de l'Inra, notamment des départements "Génétique animale " et "Physiologie animale et systèmes d'élevage " en partenariat avec les interlocuteurs de la filière bovine française et de nombreux laboratoires européens. 


\section{Petit glossaire pour décrypter ce dossier génomique}

${ }^{1}$ Génomique : science qui étudie le génome. II s'agit, pour un organisme donné, de faire à la fois l'inventaire de l'ensemble de ses gènes, de les localiser sur les chromosomes, de caractériser leur séquence, et enfin d'étudier leur(s) fonction(s).

Gène : segment d'acide nucléique à localisation précise (locus) sur un chromosome, porteur d'une information génétique utilisée par la cellule pour élaborer les protéines.

Chromosome : élément du noyau des cellules, formé d'une longue molécule d'acide nucléique (ADN) associée à des protéines. Les chromosomes contiennent à l'état condensé la double hélice d'ADN qui se dédouble lors de la division cellulaire.

Séquence : succession d'unités constitutives d'une molécule. Par exemple, la succession des nucléotides définit la séquence des acides nucléiques.

Information génétique : instructions héréditaires qui participent à l'élaboration d'un phénotype.

Acides nucléiques: molécules fondamentales pour le stockage, le maintien et le transfert de l'information génétique. La donnée indispensable pour construire les protéines et permettre la réplication d'une cellule ou d'un organisme est généralement portée par les acides désoxyribonucléiques, ou ADN, localisés au niveau des chromosomes. Lors de la transcription, l'information portée par un gène est copiée sous forme d'une molécule d'acide ribonucléique, ou ARN, dénommée ARN messager (ARNm). Cette molécule d'ARN est ensuite traduite en une protéine, le code de traduction (encore appelé code génétique) étant constitué par I'enchaînement de trois nucléotides de I'ARNm ou codon. Chaque acide aminé est codé par un ou plusieurs codons spécifiques. La traduction fait intervenir d'autres types d'ARN, les ARN de transfert (ARNt), chargés de transporter chaque acide aminé sur le lieu de synthèse de la protéine, et les $A R N$ ribosomaux ( $A R N r)$, constituant des ribosomes qui sont les organites cellulaires sur lesquels se déroule cette synthèse.

Chaque nucléotide comprend une molécule de sucre (le désoxyribose pour l'ADN et le ribose pour l'ARN) porteuse d'un groupe phosphate et d'une base azotée. Les bases azotées sont de type purique (adénine A et guanine G) ou pyrimidique (cytosine $\mathrm{C}$ et thymine T dans I'ADN, cytosine et Uracile $U$ dans I'ARN). Les molécules d'ARN ont en général une seule chaîne de nucléotides. L'ADN est constitué le plus souvent de deux chaînes (ou brins) maintenues par des liaisons hydrogène entre bases azotées : A est toujours apparié à T et G à C. Les deux brins complémentaires, enroulés en double hélice, se séparent lors de la réplication des cellules et servent de modèle pour la synthèse d'un nouveau brin complémentaire. Ce mécanisme permet aux cellules mères de transférer aux cellules filles l'information génétique contenue dans l'enchaînement des nucléotides. Ces brins se séparent aussi lors de la transcription d'ADN ou ARN, un seul brin (codant) étant transcrit en ARNm complémentaire. Des erreurs ou mutations peuvent se produire au cours de la réplication. Ces mutations, ponctuelles (au niveau d'un nucléotide) ou chromosomiques (modification plus importante sur un chromosome), sont responsables de l'évolution des êtres vivants et de pathologies (maladies génétiques, cancer). Les agents mutagènes (rayonnements UV, molécules toxiques, etc.) peuvent induire ces mutations.

Acide aminé : élément constitutif des protéines. II y a vingt acides aminés codés par un système de trois nucléotides (codons), dans I'ADN et I'ARN.

${ }^{2}$ Séquençage : technique qui consiste à déterminer l'ordre de succession des composants d'une molécule (par exemple les bases constitutives d'une molécule d'ADN).

${ }^{3}$ Puce à ADN : support miniature sur lequel sont déposés en grand nombre des fragments d'ADNc (ou microréseau d'ADNc) et permettant, par la technique d'appariement par complémentarité, de comparer la séquence ou le niveau d'expression d'un très grand nombre de gènes issus d'échantillons biologiques différents. Aujourd'hui, les ADNc sont de plus en plus souvent remplacés par des oligonucléotides de synthèse, spécifiques de chaque gène.

${ }^{4}$ Métabolite : substance issue de la transformation d'une matière organique.

${ }^{5}$ Polymorphisme : coexistence au sein d'une population de différentes formes alléliques pour des gènes ou des marqueurs. Allèle : forme que peut prendre un gène ou un marqueur génétique au niveau de la séquence d'ADN. Dans toutes les cellules somatiques, il y a deux chromosomes et donc deux allèles pour chaque gène et chaque marqueur. Si ces deux allèles sont identiques, l'individu portant le gène (ou le marqueur) est dit homozygote. Si les deux allèles diffèrent, l'individu portant le gène (ou le marqueur) est dit hétérozygote.

${ }^{6}$ Expression des gènes : étapes successives entre le gène et les protéines, incluant notamment la transcription et la traduction. Transcription: synthèse d'une molécule d'ARN (ARNm ou ARN messager), complémentaire d'une séquence d'ADN.

Traduction : synthèse de protéine à partir d'un ARNm.

7 Transcrit : ARNm résultant du processus de transcription. Le plus souvent, les ARNm d'un échantillon sont convertis dans les laboratoires en $A D N$ complémentaires (ADNc), double brins, plus appropriés pour les travaux de recherche.

8 Phénotype: ensemble des caractères réalisés et observables chez un individu. Le phénotype varie en fonction des caractéristiques héréditaires (génotype) et des effets du milieu (maîtrisés ou non par l'éleveur) qui modulent l'expression du génome.

9 Génotype : ensemble des caractéristiques héréditaires d'un individu. En effet, le patrimoine génétique (génome) de chaque animal est différent, et le génotype correspond à la combinaison des allèles qu'il possède pour chaque gène ou marqueur.

${ }^{10}$ Spectrométrie de masse : méthode d'analyse quantitative et qualitative des constituants d'un échantillon (plasma, cellule, etc.) fondée sur la séparation de ces derniers en fonction de leur masse et de leur charge après ionisation et parfois fragmentation. 


\section{Références}

Bernard C, Degrelle S, Ollier S, et al. cDNA macro-array resource for gene expression profiling in ruminant tissues involved in reproduction and production (milk and beef) traits J Physiol Pharmacol 2005 ; 56 (Supp. 3) : 215 24

Canales RD, Luo Y, Willey JC, et al. Evaluation of DNA microarray results with quantitative gene expression platforms. Nat Biotechnol gene expression plat $2006 ; 24: 1115-22$.

Cho KK, Han KH, Kang SK, Lee SH, Choi YJ. Applications of cDNA microarray in ruminants. Proceedings of the $4^{\text {th }}$ Korea-Japan Joint Symposium on Rumen Metabolism and Physiology, May 21-24, 2002, Jeju, Korea. http ://rumen.snu.ac.kr/symposium/Lectures/ Cho_KK.pdf.

Cribiu EP, Schibler L. Introduction à la cartographie des génomes complexes. INRA Prod Anim 2000 ; (Hors série) : 69-72.

Fox Keller E. Génome, postgénome : quel avenir pour la biologie? La Recherche 2004 (376) : 30-7.

Gibbs R, Weinstock G, Kappes S, Loren S, Womack J. White paper on bovine genomic sequencing initiative. 2004. (http ://www. genome.gov/Pagfes/research/Sequencing/ Senome.gov/Pagfes/research/S
Harlizius B, van Wijk R, Merks JWM. Genomics for food safety and sustainable anima production. J Biotechnol $2004 ; 113: 33-42$.

Hayes H. ADN et chromosomes. INRA Prod Anim 2000 ; (Hors série) : 13-20.

Hocquette JF, Lehnert S, Reecy J, CassarMalek I, Martin P. cDNA arrays for gene expression profiling in cattle. Proceedings of the $2^{\text {nd }}$ International Conference on Cybernetics and Information Technologies, Systems and Applications, Volume 1, Orlando (Florida, USA) July 14-17, 2005.

Hocquette JF. Where are we in genomics? J Physiol Pharmacol 2005 ; 56 (Supp 3) : 37-70.

Lehnert SA, Wang YH, Byrne KA. Development and application of a bovine cDNA microarray for expression profiling of muscle and adipose tissue. Aus J Exp Agr 2004; 44 1127-33.

Leroux C, Tosser-Klopp G. La fonction du gène: le grandes étapes de l'utilisation de I'information génétique. INRA Prod Anim 2000 ; (Hors série) : 21-8.

Liinamo AE, Neeteson-van Nieuwenhoven AM. The economic value of livestock production in the European Union. Farm Animal Industrial Platform (FAIP). Oosterbeek (The Netherlands) : AnNe Publishers, 2003.

Moody DE, Rosa AJ, Reecy JM. Current status of livestock DNA microarrays. AgBiotechNet $2003 ; 5: 1-8$.
Picard B, Lefaucheur L, Berri C, Duclos MJ. Muscle fibre ontogenesis in farm animal species. Reprod Nut Dev 2002 ; 42 : 415-31.

Rechenmann $\mathrm{F}$, de Jong $\mathrm{H}$. Le vivant en équations. La Recherche 2005 ; (383) : 32-7.

Rosati A, Tewolde A, Mosconi C. Animal production and animal science worldwide. World Association for Animal Production, Book of the Year. Wageningen (The Netherlands): Wageningen Academic Publishers, 2005.

Shi L, Reid LH, Jones WD, et al. The MicroArray Quality Control (MAQC) project shows inter- and intraplatform reproducibility of gene expression measurements. Nat Biotechnol 2006 ; 24 : 1151-61.

Smith TPL, Grosse WM, Freking BA, et al. Sequence evaluation of four pooled-tissue normalized bovine cDNA libraries and construction of a gene index for cattle. Genome Res $2001 ; 11: 626-30$.

Suchyta SP, Sipkovsky S, Kruska R, et al. Development and testing of a high-density cDNA microarray resource for cattle. Physiol Genomics 2003 ; 15 : 158-64.

Sudre K, Leroux C, Cassar-Malek I, Hocquette JF, Martin P. A collection of bovine cDNA probes for gene expression profiling in muscle. Mol Cell Probes $2005 ; 19: 61-70$.

Tuggle CK, Dekkers JCM, Reecy JM. Integration of structural and functional genomics. Anim Genet 2006 ; 37 (Suppl.1) : 1-6. 